\title{
Performance Improvement of OOFDM Systems Based On Advanced Logarithmic Companding Technique
}

\author{
${ }^{1}$ Ali N. Kareem, ${ }^{2}$ Sinan M. Abdul Satar, ${ }^{3}$ Mohammed A. Husein, ${ }^{4}$ Liqaa A. Al-Hashime, \\ ${ }^{5}$ Ghaida A. Al-Suhail \\ ${ }^{1,2,3}$ Department of Electrical Engineering, University of Technology, Baghdad, Iraq; \\ ${ }^{4,5}$ Department of Electrical Engineering, University of Basra, Basra, Iraq; \\ alinkareem@yahoo.com; sinansma@yahoo.com; mfzay@yahoo.com; leqaa.abdulsattar@gmail.com; \\ ghaida_alsuhail@yahoo.com;
}

\begin{abstract}
High peak-to-average power ratio (PAPR) is a common problem in Orthogonal Frequency Division Multiplexing (OFDM) transmissions. In this paper, an advanced logarithmic companding technique is interested to reduce this factor due to their flexibility and low complexity. We evaluate the performance of the proposed advanced logarithmic technique comparing with typical logarithmic and un-companded schemes via simulations in Intensity Modulation/Direct Detection Optical Orthogonal Frequency Division Multiplexing (IM/DD OOFDM) system. The proposed advanced logarithmic Companding technique guarantees the improved performance in terms of Bit Error Rate (BER) and Quality Factor (QF) while reducing PAPR effectively and efficiently by modifying the amplitude of the transmitted signals. Our results confirm that the suggested scheme exhibits a good ability to reduce PAPR and a good BER performance based on the use the both of ( $k$ and $y$ ) factors to be chosen in relation to acceptable or desired PAPR, BER, and QF requirements. At the complementary cumulative distribution function (CCDF) of $10^{-3}$, the PAPR value of our proposed scheme is about $6.4 \mathrm{~dB}$ lower than those of un-companded signal at best control factor used of $y=0.1$, accordingly the $Q F$ is $11.8 \mathrm{dBm}$ and the BER is $4.9 \times 10^{-5}$.

Keywords: Peak-to-Average Power Ratio (PAPR), Orthogonal Frequency Division Multiplexing (OFDM), Intensity Modulation/Direct Detection Optical Orthogonal Frequency Division Multiplexing (IM/DD OOFDM), Quality Factor (QF), Bit Error Rate (BER), Complementary Cumulative Distribution Function (CCDF).
\end{abstract}

\section{Introduction}

An Orthogonal Frequency Division Multiplexing (OFDM) has been using in the optical communication systems because of the following advantages: immunity to Inter-symbol Interference (ISI), high spectral efficiency, co-channel interference and impulsive parasitic noise, lower implementation complexity in comparison with the single carrier solution [1, 2]. On the logical ground, the intensity Modulation/Direct Detection Optical OFDM (IM/DD OOFDM) system has been widely investigated in high-speed optical Communications, short-range as well as the cost-sensitive [3]. It has been illustrated that in addition to their many advantages, OOFDM systems have also several disadvantages such as ISI and Peak-to-Average 
Ali n. Kareem, Sinan M. Abdul Satar, Mohammed A. Husein, Liqaa A. Al-Hashime, Ghaida A. Al-Suhail; Performance Improvement Of OOFDM Systems Based On Advanced Logarithmic Companding Technique Transactions on Networks and Communications, Volume 6 No 5, October (2018); pp: 121-130

Power Ratio (PAPR), which will be focused in this paper. Moreover, the high peaks to average power ratio set high power as an input to the Power Amplifier at the transmitter [2].

The high power amplifier (HPA) is working in the nonlinear section, therefore, In-band and out-of-band interferences are increased due to this nonlinearity. Reducing the PAPR will enhance HPA performance, reduce power consumption, reduce signal distortion by HPA and improve bit error ratio (BER) performance. The performance of the transmitter can be enhanced by using PAPR reduction techniques [4].

Multiple PAPR reduction techniques are offered for multi-carrier systems such as Clipping and predistortion [5-7], Partial Transmit Sequence, Selective Mapping [8-10], nonlinear companding [11-13], Tone Injection, Tone Reservation [14-16], etc. on all those PAPR reduction systems, the increasing in bandwidth and large memory with complexity in these techniques, so that the clipping and Companding technique is useful for PAPR to overcome this constraint [4, 5].

The clipping process is entirely the easiest system to use but this processing leads to distortions and causes an increase in the BER of the system [7]. Therefore, The other way is using the companding techniques that give better performance than clipping techniques due to the fact that the companding transformation is done at the transmitter to attenuate the high peaks, where as an increase low peaks and an inverse companding transform is done on the receiver end to reduce the distortion of signals and pick up the original signal, before transmission [6] [11].

The OOFDM signal involves severally modulated subcarriers that may provide a large PAPR once valueadded up coherently. An OOFDM signal features a massive PAPR that is terribly sensitive to the nonlinearity of the high peak [3]. To illustrate this point, blocks of the symbol in the OFDM are selected with every symbol modulating one from a set of subcarriers and these subcarriers are recognized to be orthogonal [8]. The representation of complex OFDM signals is given as:

$$
\mathrm{x}(\mathrm{K})=\frac{1}{\mathrm{~N}} \sum_{\mathrm{n}=0}^{\mathrm{N}-1} X_{\mathrm{n}} \mathrm{e}^{\frac{\mathrm{j} 2 \pi \mathrm{nk}}{\mathrm{N}}}
$$

Where:

$\mathrm{K}=0,1,2, \ldots, \mathrm{N}-1$

\section{The Peaks to Average Power Ratio (PAPR)}

The PAPR for a signal $x(K)$ is outlined as the ratio of maximum instantaneous power to the average power as illustrated in (2) [3]:

$$
\operatorname{PAPR}=10 * \log \left(\frac{\max |\mathrm{x}(\mathrm{K})|^{2}}{\operatorname{Avg}|\mathrm{x}(\mathrm{K})|^{2}}\right)
$$

Furthermore, the reduction of PAPR simply shows the probabilities that the PAPR of data block exceeds a given threshold value. The expression of Complementary Cumulative Distribution Function (CCDF) is given in (3) (Akhtman et al.,2003)[2]:

$$
\text { CCDF=Probability(PAPR >PAPRth) }
$$

Where PAPRth represents the Threshold level. 


\section{Advanced Logarithmic Companding Technique}

It has been noticed that Due to low complexness regardless of the number of subcarriers, the Companding technique has been using for PAPR reduction in OFDM systems [2]. Moreover, the logarithmic transform using at the transmitter as a compressor after Inverse Fast Fourier Transform (IFFT) that is described in equation (4) and an expander before Fast Fourier Transform (FFT) at the receiver that described in equation (5):

$$
\begin{aligned}
& f(x)=\log (1+(K *|x|)) * \operatorname{sgn}(x) \\
& f(x)^{-1}=\left|\left(\exp \left(\frac{|x|}{K}\right)-1\right)\right| * \operatorname{sgn}(x)
\end{aligned}
$$

Where:

sgn: sign function

$\mathrm{k}$ : is a positive number for controlling the amount of companding.

Additionally, a new modification of the logarithmic technique has been proposed depending on the original equation by proposing a new factor $(y)$ that's also controlling the amount of companding as well as maintain the input and output signals at the same average power level. The advanced companded function is given in equation (6) and equation (7):

$$
\begin{aligned}
& \mathrm{f}(\mathrm{x})=\log \left(1+(\mathrm{K} *|\mathrm{x}|)^{\mathrm{y}}\right) * \operatorname{sgn}(\mathrm{x}) \\
& \mathrm{f}(\mathrm{x})^{-1}=\left|\left(\exp \left(\frac{|\mathrm{x}|}{\mathrm{K}}\right)-1\right)^{1 / \mathrm{y}}\right| * \operatorname{sgn}(\mathrm{x})
\end{aligned}
$$

Where:

y: positive number $(1,0.9, \ldots, 0.1)$

\section{The Proposed Modeling OOFDM System}

Firstly, the traditional configuration of the OOFDM system without using the companded technique is shown in figure 1 .

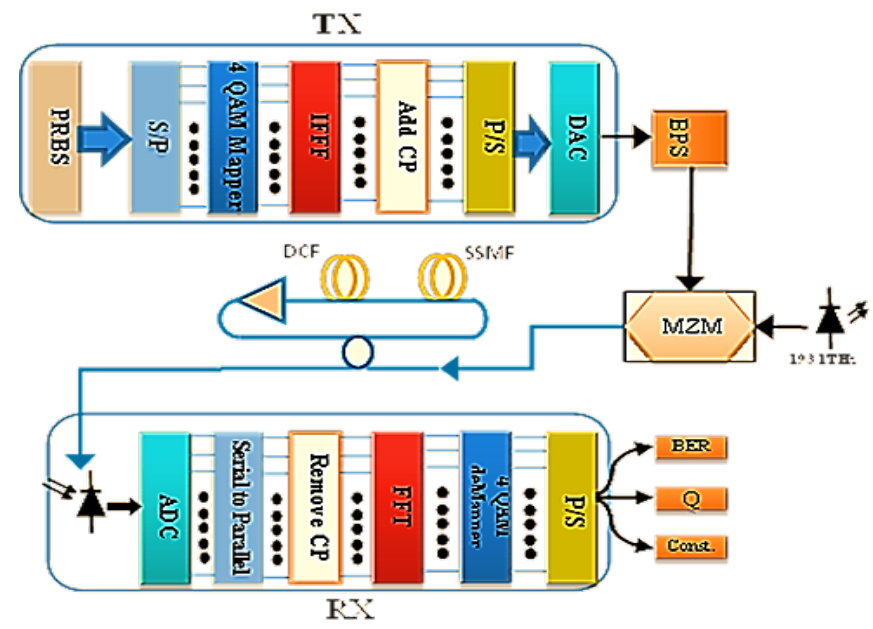

Figure 1. The traditional OOFDM system. 
Ali n. Kareem, Sinan M. Abdul Satar, Mohammed A. Husein, Liqaa A. Al-Hashime, Ghaida A. Al-Suhail; Performance Improvement Of OOFDM Systems Based On Advanced Logarithmic Companding Technique Transactions on Networks and Communications, Volume 6 No 5, October (2018); pp: 121-130

The proposed modeling system by using an advanced logarithmic companding technique is shown in figure 2 and compared with the traditional system, the logarithmic transform applied in transmitter and receiver as shown in figure 2 after Fourier in the transmitter and before Fourier in the receiver.

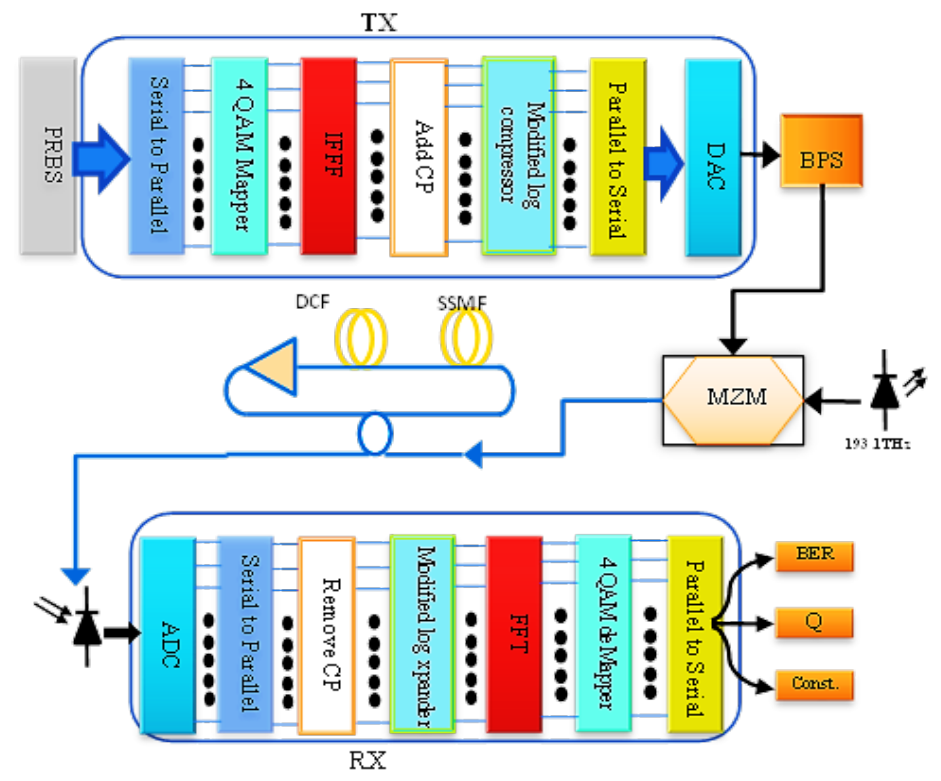

Figure 2. The proposed OOFDM System with advanced logarithmic transform.

As far as it is concerned, The transmitted digital bit streams are originated in VPI Transmission Maker software package from the Pseudorandom binary sequence (PRBS) at $\left(2^{13}-1\right)$ and then OFDM data are decoded with and without logarithmic transform by MATLAB software package. VPI Transmission Maker does the optical modulation and optical up-down conversion and transmission link. The companded signal transmitted through the optical link with length equal to $900 \mathrm{Km}$ (60 Km $\times 15$ loops). The Fiber link contains a Standard Single Mode Fiber (SSMF), a Dispersion Compensation Fiber (DCF), and an optical amplifier. In all as feds, the SSMF is $50 \mathrm{~km}$ length with $0.2 \mathrm{~dB} \cdot \mathrm{km}^{-1}$ attenuation coefficient and $16 \mathrm{ps} \cdot \mathrm{nm}^{-1} \cdot \mathrm{km}^{-1}$ of Chromatic Dispersion (CD) coefficient. For compensating the fibers losses, the optical amplifier has gain and noise figure of $12 \mathrm{dBm}$ and $4 \mathrm{dBm}$ respectively. Finally, in the receiver, the reverse operation occurs after detection to restore to the original signal.

\section{Results and Discussion}

The coded OFDM signal (real and imaginary part of companded OFDM) is up-converted at $7.5 \mathrm{GHz}$ an Intermediate Frequency (IF) as shown in figure 3. 


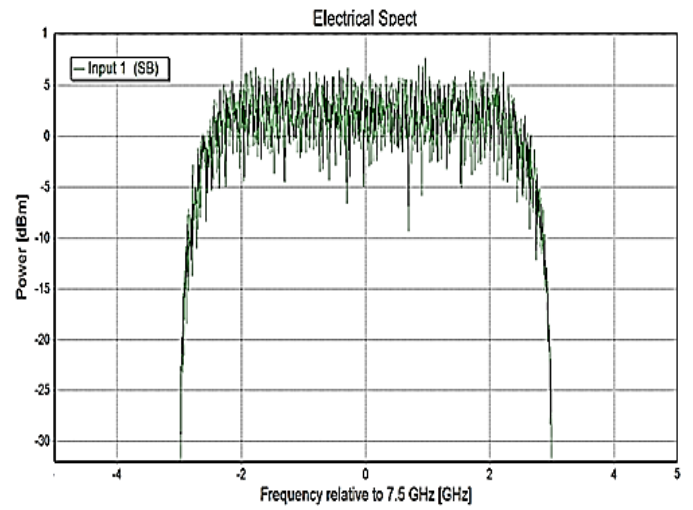

Figure 3. Electrical Spectrum of proposed logarithmic OOFDM at IF=7.5 GHz.

The output spectrum power from MZM contains an optical carrier that is targeted at $193.1 \mathrm{THz}$ in this case and two side-bands targeted at $7.5 \mathrm{GHz}$ of the optical OFDM signal as shown in figure 4.

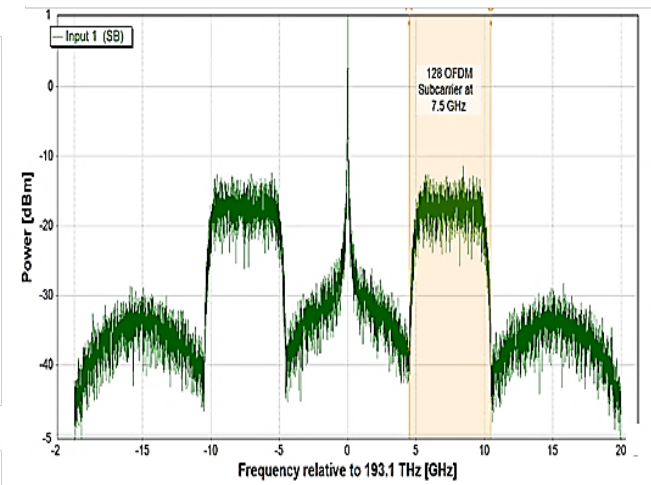

Figure 4. The spectrum of the proposed advanced logarithmic OOFDM.

Likewise, an optical filter is then responsible for suppressing the lower sideband by $18 \mathrm{GHz}$ bandpass optical filter, the filtered advanced OOFDM single shows in figure 5.

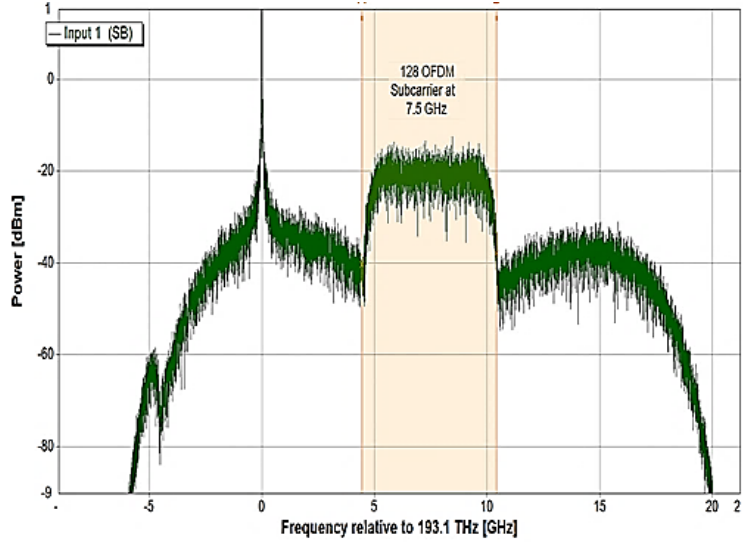

Figure 5. The spectrum of the proposed advanced logarithmic OOFDM after Filtering.

Figure 6 summarizes the performance of various degree $(y)$ with the PAPR of OFDM companded signals. The PAPR values for degrees larger than 0.2 are almost the same as that for less than 0.15 . Moreover, the 
Ali n. Kareem, Sinan M. Abdul Satar, Mohammed A. Husein, Liqaa A. Al-Hashime, Ghaida A. Al-Suhail; Performance Improvement Of OOFDM Systems Based On Advanced Logarithmic Companding Technique Transactions on Networks and Communications, Volume 6 No 5, October (2018); pp: 121-130

minimum PAPR must be obtained at this range where $0.2 \leq \mathrm{y} \leq 0.15$ due to these values add more compression at the transmitter's side system.

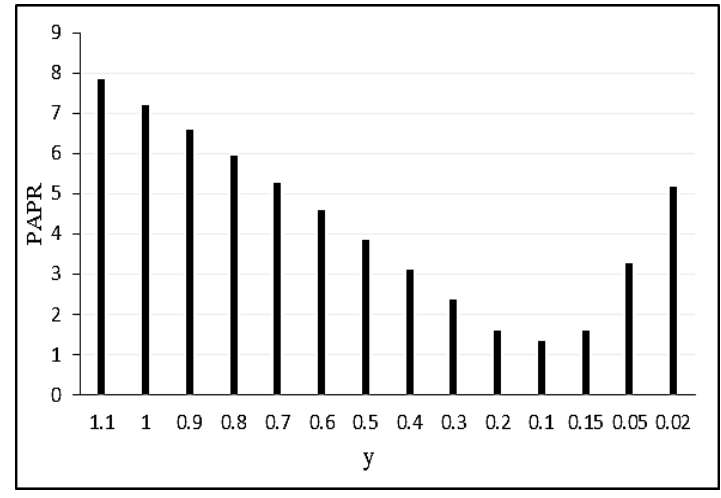

Figure 6. PAPR reduction vs control degree y.

The PAPR performance of the OOFDM transmitter based on original logarithmic technique is significantly improved. The PAPR with typical logarithmic technique is reduced while the controlling factor $(k)$ is increased as shown in figure 7.

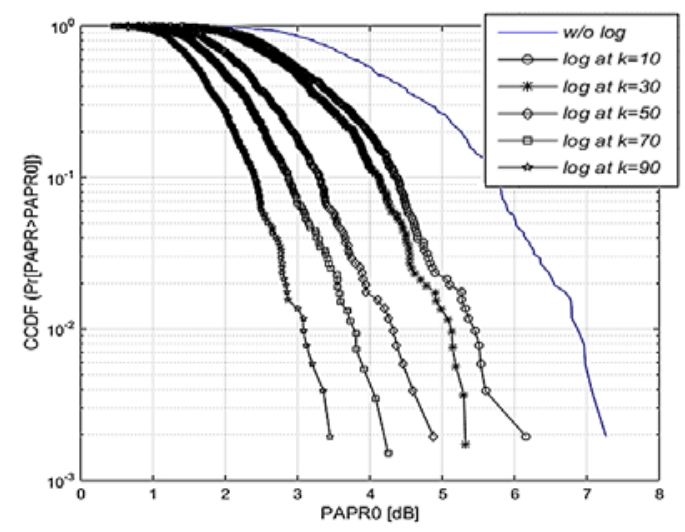

Figure 7. CCDF plot of typical logarithmic PAPR reduction technique

The PAPR reduction based on an advanced logarithmic technique depends on the two controls $k$ and $y$ as shown in figure 8 and figure 9. The CCDF is often used to display the PAPR probability distributions of OFDM transmissions. CCDF allow the probability of PAPR exceeding any given value of PAPR to be determined. From figure $8(\mathrm{a})$ and $8(\mathrm{~b})$ it is seen that the PAPR of the companded OFDM signal is much lower than the un-companded signal. Companding the 128-subcarrier signal with $k=30$ and 50 , gives the PAPR as $1.5 \mathrm{~dB}$ for $\mathrm{k}=30$, which increases to $2.5 \mathrm{~dB}$ for $\mathrm{k}=50$ where $\mathrm{y}=1$. It may be noted that the reduction in PAPR is smaller for higher values of $k$. 


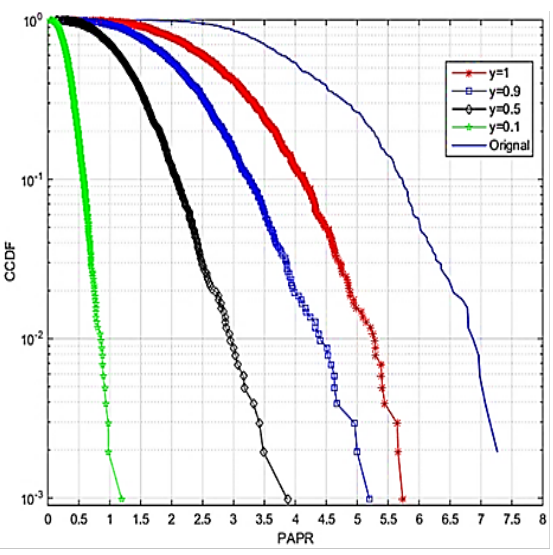

(a)

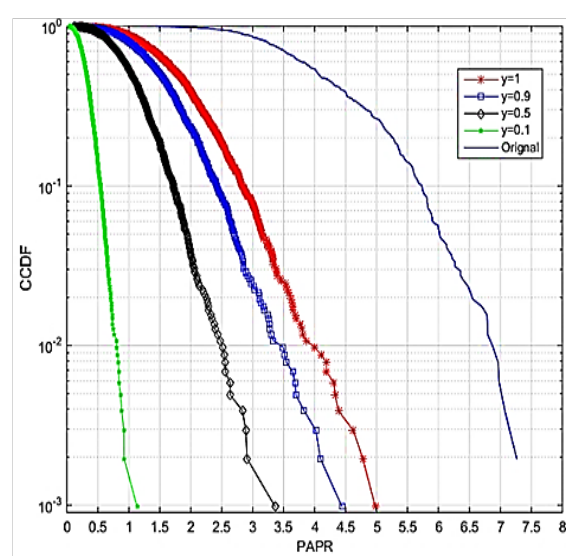

(b)

Figure 8. CCDF plot vs PAPR based on advanced logarithmic technique for different control degree y where (a) $k=30$ (b) $k=50$

Figure 9 shows the CCDF performance with companding degree $y=1$ and 0.1 , it can be noted that $y=0.1$ provides a slightly better performance than $\mathrm{y}=1$ for all values of $\mathrm{k}$.

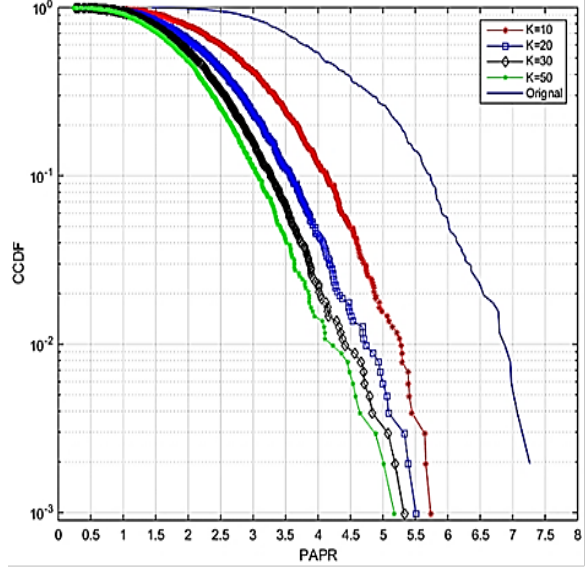

(a)

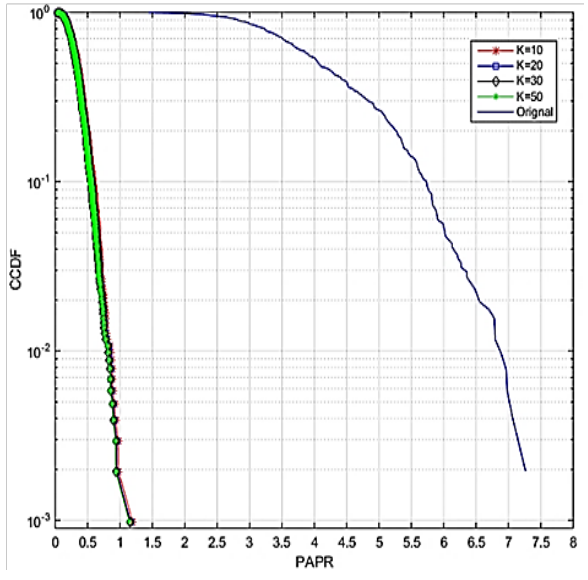

(b)

Figure 9. CCDF plot vs PAPR based on advanced logarithmic for different control factor $\mathrm{k}$ where (a) $y=1$ (b) $y=0.1$

After the fiber length at $900 \mathrm{Km}(60 \mathrm{Km} \times 15)$ with 4 QAM modulation formats The Quality Factor (QF), BER and constellation diagram at the receiver end is shown in figure 10, figure 11 and figure 12 respectively. Moreover, the degradation observed in the original system without companded technique used is shown in figure 10 , figure 11 plotted without a marker, the measured QF is $4.8 \mathrm{dBm}, \mathrm{BER}$ is $1.58 \times 10^{-}$ 3 and non-uniform constellation diagram in figure 12(a). This degradation is considered the maximum because of the increase in the chromatic dispersion when increasing the transmission length.

Mostly, the proposed advanced logarithmic transform that plotted with marker red color clearly improves the OOFDM system performance that is described as QF, BER, and constellation. From figure 10, figure 11 and figure 12, two values of $y$ at 1 and 0.1 are proposed, the value of $y=0.1$ is totally enhancing the all 
Ali n. Kareem, Sinan M. Abdul Satar, Mohammed A. Husein, Liqaa A. Al-Hashime, Ghaida A. Al-Suhail; Performance Improvement Of OOFDM Systems Based On Advanced Logarithmic Companding Technique Transactions on Networks and Communications, Volume 6 No 5, October (2018); pp: 121-130

system in terms of QF at $12 \mathrm{dBm}$ and $B E R$ at $4.8 \times 10^{-5}$ than that is using $y=1$ which $Q F=8.8 \mathrm{dBm}$ and $B E R$ $=4.96 \times 10^{-4}$ at distance link $=900 \mathrm{~km}$

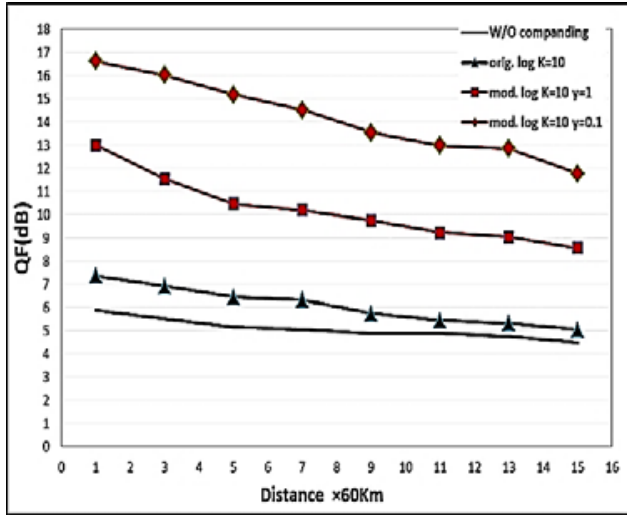

Figure 10. QF vs Transmission length

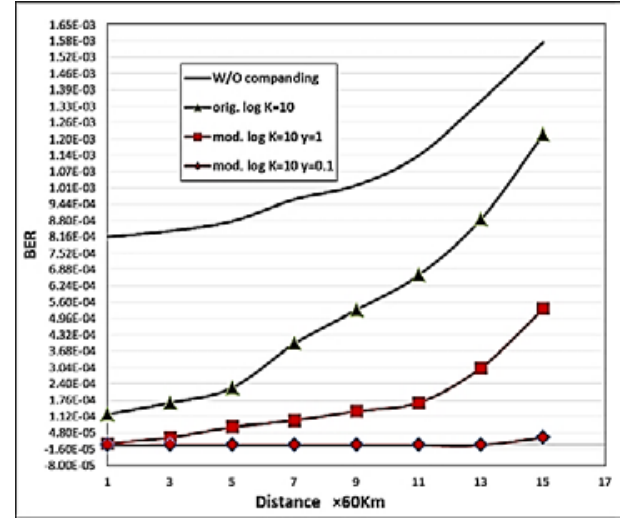

Figure 11. BER vs Transmission Length

Clearly as shown in figure 12 , companding with $y=1$ provides a degraded constellation diagram, however, with $y=0.1$ a significant improvement in constellation performance over without companded or companded at $y=1$.

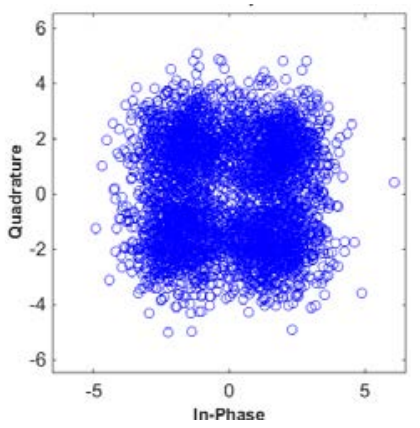

(a)

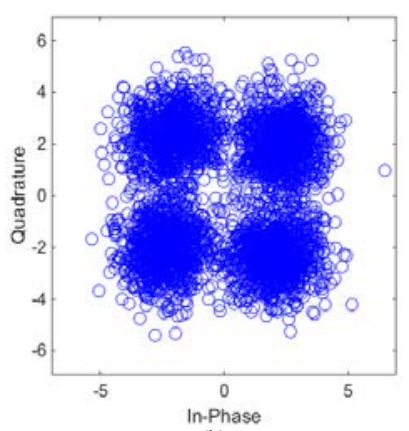

(b)

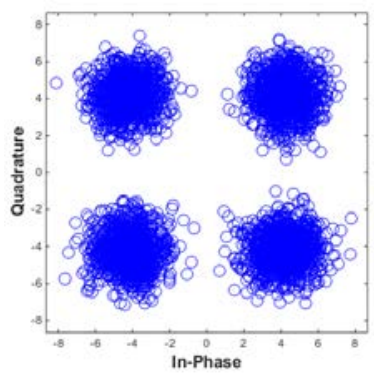

(c)

Figure 12. Constellation diagram of advanced OOFDM system at the receiver end after $900 \mathrm{Km}$ at (a) original without companded (b) Proposed companded at $\mathrm{y}=1$ and $\mathrm{k}=10$ (c) Proposed companded at $\mathrm{y}=0.1$ and $\mathrm{k}=10$.

\section{Conclusion}

The proposed IM/DD OOFDM transmitter system based on an advanced logarithmic companding technique has been implemented in order to reduce the PAPR of OOFDM systems implemented based on the sequence of operation shown in figure 2. Moreover, the results show the $y=0.1$ is a best one to reduce the PAPR system performance instead of any value of $k$. All results of $Q F, B E R$ and constellation have been presenting by combination VPI Transmission Maker with MATLAB software package. In future, the 
performance of transceiver systems can be completely more improved by proposing a new value for $\mathrm{k}$ and y with combination another technique.

\section{REFERENCES}

[1] R. v. Nee and R. Prasad, OFDM for Wireless Multimedia Communications: Artech House, Inc., 2000.

[2] J. Akhtman, B. Z. Bobrovsky, and L. Hanzo, "Peak-to-average power ratio reduction for OFDM modems," in The 57th IEEE Semiannual Vehicular Technology Conference, 2003. VTC 2003-Spring., vol. 2, pp. 11881192 vol.2, 2003.

[3] X. Zhang, P. Liu, J. Liu, and S. Liu, "Advanced A-law employing nonlinear distortion reduction in DCO-OFDM systems," in 2015 IEEE/CIC International Conference on Communications in China - Workshops (CIC/ICCC), pp. 184-188, 2015.

[4] J. Zhou and Y. Qiao, "Low-PAPR Asymmetrically Clipped Optical OFDM for Intensity-Modulation/DirectDetection Systems," IEEE Photonics Journal, vol. 7, no. 3, pp. 1-8, 2015.

[5] V. Cuteanu and A. Isar, "PAPR reduction of OFDM signals using hybrid clipping-companding scheme with sigmoid functions," in 2011 International Conference on Applied Electronics, pp. 1-4, 2011.

[6] A. N. D. Andrea, V. Lottici, and R. Reggiannini, "Nonlinear predistortion of OFDM signals over frequencyselective fading channels," IEEE Transactions on Communications, vol. 49, no. 5, pp. 837-843, 2001.

[7] J. Armstrong, "Peak-to-average power reduction for OFDM by repeated clipping and frequency domain filtering," Electronics Letters, vol. 38, no. 5, pp. 246-247, 2002.

[8] T. Jiang and Y. Wu, An Overview: Peak-to-Average Power Ratio Reduction Techniques for OFDM Signals vol. 54, 2008.

[9] L. Wang and J. Liu, "PAPR Reduction of OFDM Signals by PTS With Grouping and Recursive Phase Weighting Methods," IEEE Transactions on Broadcasting, vol. 57, no. 2, pp. 299-306, 2011.

[10] S. Y. L. Goff, B. K. Khoo, C. C. Tsimenidis, and B. S. Sharif, "A novel selected mapping technique for PAPR reduction in OFDM systems," IEEE Transactions on Communications, vol. 56, no. 11, pp. 1775-1779, 2008.

[11] Y. Wang, J. Ge, L. Wang, J. Li, and B. Ai, "Nonlinear Companding Transform for Reduction of Peak-toAverage Power Ratio in OFDM Systems," IEEE Transactions on Broadcasting, vol. 59, no. 2, pp. 369-375, 2013.

[12] K. Anoh, B. Adebisi, K. M. Rabie, and C. Tanriover, "Root-Based Nonlinear Companding Technique for Reducing PAPR of Precoded OFDM Signals," IEEE Access, vol. 6, pp. 4618-4629, 2018.

[13] S. Azou, S. Bejan, P. Morel, and A. Sharaiha, "A comparative study of nonlinear companding schemes for CO-OFDM transmissions," in 2014 13th International Conference on Optical Communications and Networks (ICOCN), pp. 1-4, 2014. 
Ali n. Kareem, Sinan M. Abdul Satar, Mohammed A. Husein, Liqaa A. Al-Hashime, Ghaida A. Al-Suhail; Performance Improvement Of OOFDM Systems Based On Advanced Logarithmic Companding Technique Transactions on Networks and Communications, Volume 6 No 5, October (2018); pp: 121-130

[14] N. Jacklin and Z. Ding, "A Linear Programming Based Tone Injection Algorithm for PAPR Reduction of OFDM and Linearly Precoded Systems," IEEE Transactions on Circuits and Systems I: Regular Papers, vol. 60, no. 7, pp. 1937-1945, 2013.

[15] A. Ivanov and D. Lakontsev, "Selective tone reservation for PAPR reduction in wireless communication systems," in 2017 IEEE International Workshop on Signal Processing Systems (SiPS), pp. 1-6, 2017.

[16] W. Wang, M. Hu, Y. Li, and H. Zhang, "A Low-Complexity Tone Injection Scheme Based on Distortion Signals for PAPR Reduction in OFDM Systems," IEEE Transactions on Broadcasting, vol. 62, no. 4, pp. 948956, 2016. 